\title{
Utility of dual longitudinal diameter-reducing ties in aortic arch thoracic endovascular aortic repair
}

\author{
Arkadiusz Kazimierczak¹, Paweł Rynioํㅜ, Tomasz Jędrzejczak², Maciej Lewandowski ${ }^{3}$, Piotr Gutowski ${ }^{1}$ \\ ${ }^{1}$ Vascular Surgery Department, Pomeranian Medical University, Szczecin, Poland \\ ${ }^{2}$ Cardiac Surgery Department, Pomeranian Medical University, Szczecin, Poland \\ ${ }^{3}$ Cardiology Department, Pomeranian Medical University, Szczecin, Poland
}

Adv Interv Cardiol 2019; 15, 4 (58): 485-488

DOI: https://doi.org/10.5114/aic.2019.90224

\section{Introduction}

A 69-year-old man underwent open surgical ascending aortic replacement for type-A aortic dissection. Six months after this operation, he became symptomatic. There was persistent chest pain even though anti-hypertensive treatment was effective. Computed tomography angiography showed a widening false lumen ( $\mathrm{FL}$ ) in the aortic arch and in the thoracic aorta (Figure 1). The patient was not suitable for open aortic arch replacement due to high risk (logistic EuroSCORE 34.47\%). Standard thoracic endovascular aortic repair (TEVAR) or branched endovascular repair (e.g. COOK) [1] was not considered due to insufficient landing zone distal to a right coronary artery venous bypass graft arising from the ascending aortic graft.

\section{Case report}

\section{Stent-graft modification}

The patient was selected for endovascular treatment with a physician-modified endograft (PMEG). Due to the presence of vein coronary bypass (VCB), an endograft with four fenestrations to the innominate artery (IA), left subclavian artery (LSA), left common carotid artery (LCCA) and VCB was planned. The PMEG was prepared on the basis of $3 D$ printing $[2,3]$. Fenestrations for the IA, LCCA, LSA and VCB were marked, burnt and their edges reinforced. The LSA was pre-cannulated. The VCB fenestration, located in the proximal endograft landing zone, was extra-large and "non-sealing" in nature (Figure 1).

Due to the sharply angled aortic arch it was not possible to insert the rigid delivery system using conventional femoral access and stiff guidewire in the ascending aorta. The delivery system was more rigid because it contained a PMEG with pre-cannulation guidewire $(0.36 \mathrm{~mm} / 0.014$ inch Terumo Soft, Europe, Interleuvenlaan 403001 Leuven, Belgium) for the LSA, four fenestrations for arch vessels and VCB all marked and having reinforced edges and finally double guidewires (V-18 Control, Boston Scientific, USA) to constrain the endograft.

Therefore, externalized transapical guidewire technique (ETAG) was planned.

A Valiant Captivia thoracic endograft (Medtronic, Santa Rosa, CA, USA) of diameter $40 \mathrm{~mm}$ and length $200 \mathrm{~mm}$ was deployed in a sterile aortic arch model and after optimal positioning of the metal frame the VCB, IA, LCCA and LSA departure points were marked. Then the fenestrations were burned. Their edges were lined with the loop cut out from a snare (Indy OTW Vascular Retriever 8.0-35-55-40) for marking and strengthening the fenestration edge. The endograft was constrained on two (opposite) sides using dual longitudinal diameter-reducing ties (DLT) - each Z-stent along the endograft was constrained using a stainless steel wire (V18 Control) for support and two non-locking Prolene loops [4] (Figure 1). The LSA fenestrations were pre-cannulated using a $0.36 \mathrm{~mm} / 0.014$-inch soft Terumo guidewire fed through the delivery system, inserted from the bottom of the endograft and led out through the LSA fenestration [5]. The endograft was re-sheathed avoiding twisting within the delivery system using a transcatheter aortic valve implantation packaging device.

\section{Vascular access}

Access to the cardiac apex was obtained using a mini-thoracotomy in the 4 intercostal left space. Access to the right common femoral artery for endograft delivery system insertion was obtained by cut-down. The left 

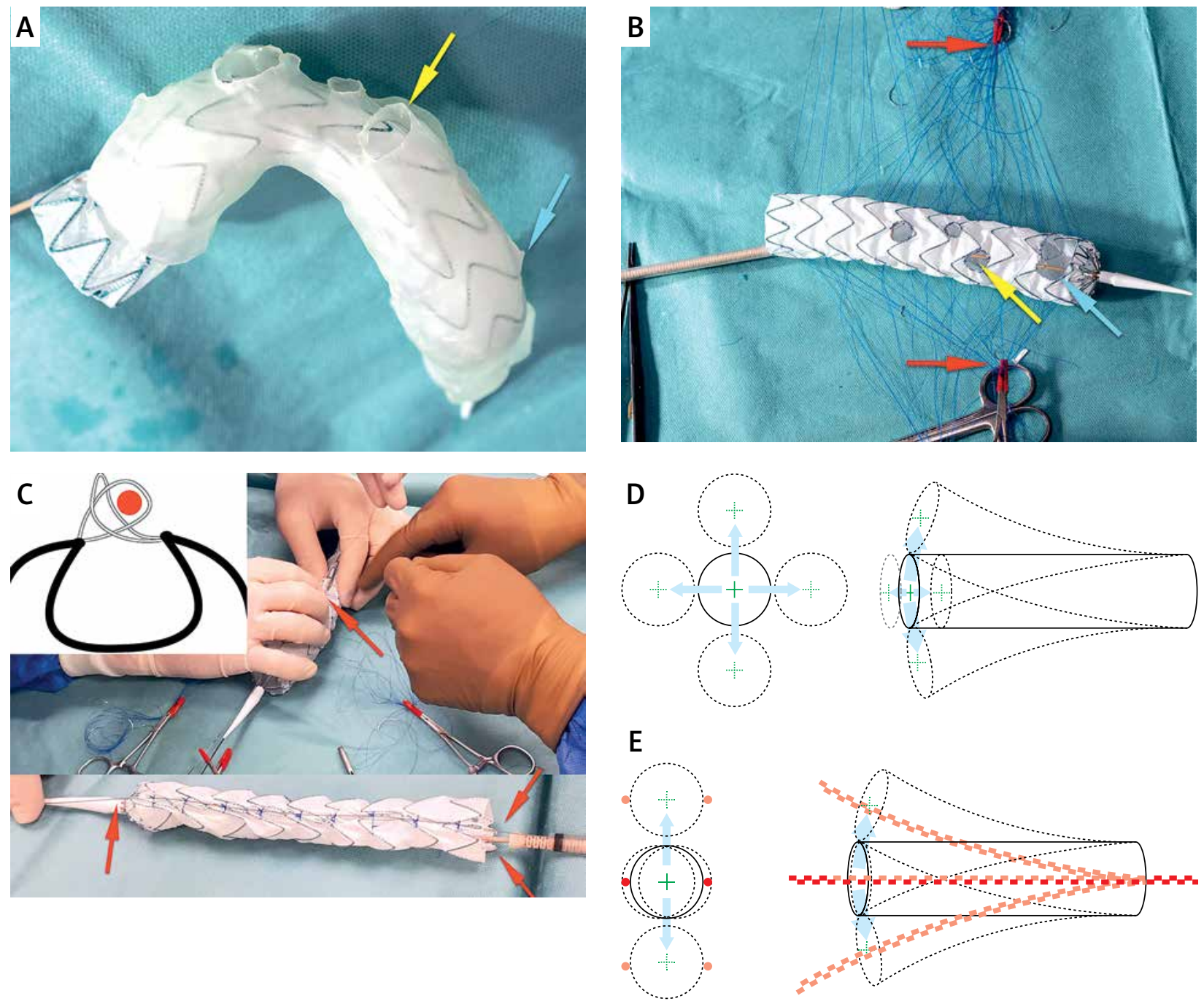

Figure 1. Principle of graft modification. A - 3D printing as a template for PMEG. B - Fenestration edge lined with the wire. PMEG ready for completing dual longitudinal diameter-reducing ties. $\mathbf{C}$ - Dual longitudinal diameter-reducing ties made with two non-locking Prolene loops one of which goes around a V18 Control wire (DLT wire shown in red). D - Delivery system without double lateral reducing ties can bend in any direction. $\mathrm{E}$ - Double lateral reducing ties limit the bending of the delivery system to only one plane. Red arrows indicate the Prolene suture needed to knot non-locking Prolene loops for dual longitudinal diameter-reducing ties. Yellow arrow indicates innominate artery. Blue arrow indicates the coronary bypass outflow

femoral artery was punctured percutaneously to introduce a $7 \mathrm{Fr}$ sheath (this access was used for LCCA stenting and inserting a pigtail diagnostic catheter into the arch). The right subclavian artery was exposed by cutdown for cannulation and stenting of the IA fenestration. Left axillary artery access was obtained by cut-down for stenting the LSA fenestration. The guidewire that passed through the LSA fenestration was exteriorized from the left axillary access prior to insertion of the arch endograft delivery system into the right femoral artery.

\section{Endograft deployment}

First, a guiding catheter passed from the left axillary artery to the right femoral artery was used to pass the
LSA pre-cannulation wire in the opposite direction. The endograft was then introduced through the same right femoral arterial access into the ascending aorta and arch using ETAG on a $0.36 \mathrm{~mm} / 0.014$-inch soft Terumo guidewire introduced from the apex to the right femoral artery under transesophageal echocardiographic and fluoroscopic monitoring.

Prior to endograft deployment, the position of all arch branches was ascertained by arteriography using a pigtail catheter inserted from left femoral arterial access. The endograft was then deployed, but it remained partially constrained because of the DLTs. Partial constraint of the endograft and traction towards the inner aortic curvature (using ETAG) were necessary to leave space for 
the cannulation of fenestrations from the side of the outer aortic arch curvature. The IA and LCCA fenestrations were cannulated (the LSA had been pre-cannulated) and covered stents positioned within all three and kept ready for deployment: BeGraft $14 \times 38 \mathrm{~mm}$ (Bentley InnoMed $\mathrm{GmbH}$, Lotzenäcker 25, 72379 Hechingen, Germany) via $12 \mathrm{Fr} / 45 \mathrm{~cm}$ guiding sheath (Flexor Ansel, COOK Medical, Bloomington, IN 47402-4195 USA) in the IA, Advanta 12 × $29 \mathrm{~mm}$ (Getinge, 40 Continental Blvd, Merrimack, NH 03054, USA) via $9 \mathrm{Fr} / 45 \mathrm{~cm}$ guiding sheath (Flexor Ansel, COOK Medical) in the LSA and BeGraft $7 \times 38 \mathrm{~mm}$ via $7 \mathrm{Fr} / 90 \mathrm{~cm}$ guiding sheath (Flexor Shuttle, COOK Medical) in the LCCA. Then both lateral ties were released and another endograft was implanted into the descending aorta (Medtronic Vailant Captiva, VAMC4238C150TE) distal to the LSA fenestration. This was done to provide a landing zone for endovascular procedures in the thoracic and abdominal aorta in the future. A tri-lobe balloon (Gore, Flagstaff, Arizona, 86003-2400 USA) was used to expand the aortic endografts and their overlap over their entire length except for the last segment of the distal endograft to avoid the phenomenon of stent-induced new entry. Afterwards the ETAG guidewire was removed from the heart. Covered stents in the fenestrations were deployed and their intra-aortic portions ( $5-8 \mathrm{~mm}$ protrusion into the aortic endograft) were flared using balloons $2 \mathrm{~mm}$ wider than the nominal stent diameters. Control arteriography confirmed the patency of the arch branch fenestrations and VCB and the lack of endoleak into the false lumen. The remaining indwelling endovascular equipment was removed and the access vessels repaired.

The course after the procedure was not complicated. The patient was discharged 10 days after the second procedure. Computed angiography tomography after 2 months showed good graft function, no endoleak, thrombosed false lumen in the arch, no stent-induced new entry below the thoracic endograft and patent VCB (Figure 2).

\section{Discussion}

Since it is not possible to rotate the endograft delivery system once it is in the aortic arch, it is necessary to enter the arch with optimal orientation. This means that the fenestrations must be directed towards the outer curvature of the aortic arch. Endograft segments have a tendency to move into each other at sharp curves, a property gainfully utilized by some endografts for better alignment to the aortic curvature (e.g. GORE TAG) [6]. The presence of rigid linear reinforcement connecting consecutive stent segments makes it impossible for the endograft to bend and concertina towards the side where the reinforcement is located and restricts bending to the opposite direction. An example is the Ankura (Lifetech, Shenzhen, China) endograft: a longitudinal supporting strut orientated to the greater curvature forces the endograft to self-orient and bend towards the side

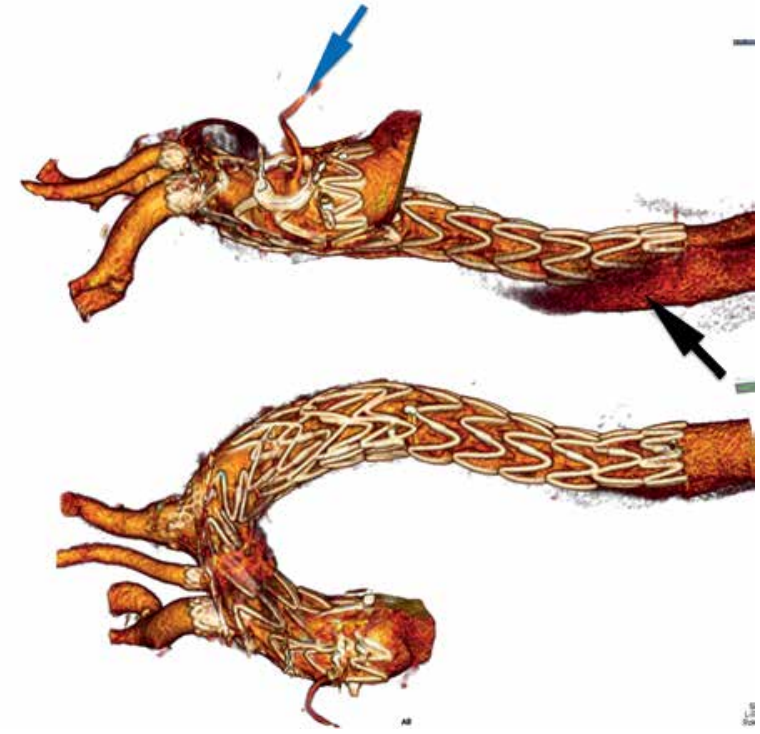

Figure 2. Good graft function. Black arrow indicates persistent flow in false lumen below the thoracic stent graft. Blue arrow indicates satisfactory positioning of the fenestration on the coronary bypass

of the lesser arch curvature naturally during insertion into the arch.

We have made use of this mechanism while making arch PMEGs. However, a single lateral tie on the outer curvature would make it impossible to cannulate fenestrations located here. Hence we used DLTs located on opposite sides of the endograft to restrict its bending to one plane only (Figure $1 \mathrm{~B}$ ). Consequently, when entering the arch, the endograft fenestrations will never orient sideways (laterally). Moreover, it allows adequate constraining of the endograft so as not to restrict blood flow in the aorta and provide sufficient space for the cannulation of fenestrations. It is expected that one diameter reducing tie will reduce the endograft diameter by about 20-30\% [4]. Attempts to increase the reduction further with a single tie can lead to endograft infolding and endoleak [7]. In our experience with the use of PMEGs in the aortic arch, as long as we used one diameter reducing tie, we seldom managed to find a suitable place for making fenestrations appropriate for the anatomy of the arch vessels. Only after using DLTs can we appropriately position fenestrations in the endograft. This technical modification has the potential to be used in all arch devices in the future.

It should be borne in mind that any manipulation of partially constrained endografts and fenestration cannulating hardware in the aortic arch (before full aortic endograft wall apposition) increases the risk of embolic stroke. Some authors prefer to use unconstrained arch endografts that appose the aortic wall completely immediately after deployment and trap arch debris underneath [8]. All this is true when the maneuvers are done with- 
out keeping the graft close to the inner arch curvature by ETAG. With ETAG the endograft is not in contact with the outer curvature of the aortic arch and the risk of stroke is lower. Moreover, all procedures in the arch are done with complete suppression of the coagulation system (ACT $>300$ s). Precise deployment of the fenestration in the arch in a one-step procedure (unconstrained endograft) is not always possible due to tortuous arch anatomy especially with two or more angulations in the arch. Therefore, having an option to cannulate misplaced fenestration (with the help of DLT and ETAG), instead of ending up with the cut-off blood flow to the brain, is a great relief. Therefore, both techniques, DLT and ETAG, could be considered simultaneously. ETAG and DLT make space available on the outer arch curvature and increase the margin for possible misplacement errors. Finally, they reduce the need for rapid cardiac pacing and facilitate passage of the endograft delivery system through sharply angled aortic arches.

It could be suggested the ETAG negates many of the advantages that percutaneous arch repair could offer. However, it is still much less invasive compared to open arch repair (no sternotomy, extra-corporal circulation and hypothermia). In the future ETAG might be even less invasive when percutaneous transapical access becomes widely available $[9,10]$.

\section{Limitations}

This is one of the first three such cases and only proves that it is possible to carry out such a procedure effectively. We do not know whether $100 \%$ repeatability of this technique is possible in all anatomical conditions. Therefore, we will collect data from subsequent treatments of this type. These are technically difficult procedures, especially in patients who have degenerating aortic arch dissection after an initially successful Bentall procedure.

\section{Conclusions}

Use of dual longitudinal diameter-reducing ties enables proper orientation of the endograft delivery system in the aortic arch. It provides enough space to manipulate the guidewires and catheters during cannulation of the fenestrations and does not restrict the blood flow inside the aorta.

\section{Conflict of interest}

The authors declare no conflict of interest.

\section{References}

1. Spear R, Clough RE, Fabre D, et al. Total endovascular treatment of aortic arch disease using an arch endograft with 3 inner branches. J Endovasc Ther 2017; 24: 534-8.

2. Rynio P, Kazimierczak A, Jedrzejczak T, Gutowski P. A 3D printed aortic arch template to facilitate decision-making regarding the use of an externalized transapical wire during thoracic endovascular aneurysm repair. Ann Vasc Surg 2019; 54: 336.e5-8.

3. Rynio P, Kazimierczak A, Jedrzejczak T, Gutowski P. A 3-dimensional printed aortic arch template to facilitate the creation of physician-modified stent-grafts. J Endovasc Ther 2018; 25: 554-8.

4. Oderich GS. Diameter-reducing wire to facilitate deployment of a modified zenith fenestrated stent graft. Ann Vasc Surg 2010; 24: 980-4.

5. Joseph G, Premkumar P, Thomson V, et al. Externalized guidewires to facilitate fenestrated endograft deployment in the aortic arch. J Endovasc Ther 2016; 23: 160-71.

6. Ito E, Kanaoka Y, Maeda K, et al. Deployment accuracy of the conformable GORE ${ }^{\circledR}$ TAG ${ }^{\circledR}$ thoracic endoprosthesis in the treatment of zones 2 and 3 aortic arch aneurysms compared with the previous TAG®. Ann Vasc Dis 2015; 8: 74-8..

7. Mirza AK, Sandri GA, Tenorio ER, et al. Severe infolding of fenestrated-branched endovascular stent graft. J Vasc Surg Cases Innov Tech 2018; 4: 240-3.

8. Joseph G. Treatment of ascending aortic pathology with arch endografts that extend proximally. J Endovasc Ther 2019; 26: 463-6.

9. Ferrari E, Berdajs D, Tozzi P, et al. Apical closure device for transapical valve procedures. Interact Cardiovasc Thorac Surg 2015; 21: 561-4.

10. Brinks H, Nietlispach F, Göber V, et al. Transapical access closure: the TA PLUG device. Interact Cardiovasc Thorac Surg 2013; 17: 806-10. 